\section{Editor-in-Chief \\ Barbara McLain - (retired Prof.) \\ University of Hawaii, USA}

The Israeli Journal of Aquaculture (IJA) is an interdisciplinary journal that is dedicated to sharing new research and tested applications of aquaculture

The IJA is devoted to scholarly articles for improved aquaculture practices and related industries

The IJA is a peer-reviewed, open-access, electronic journal

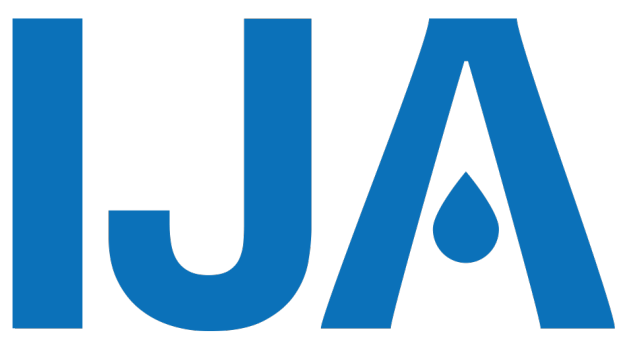

\section{The () Israeli Journal of Aquaculture}

An interdisciplinary online Open Access scientific journal

Published by the

\section{AquacultureHub}

A non-profit organization 501c3

http://www.aquaculturehub.org

in partnership with the

\section{University of Hawaii at Manoa} Library

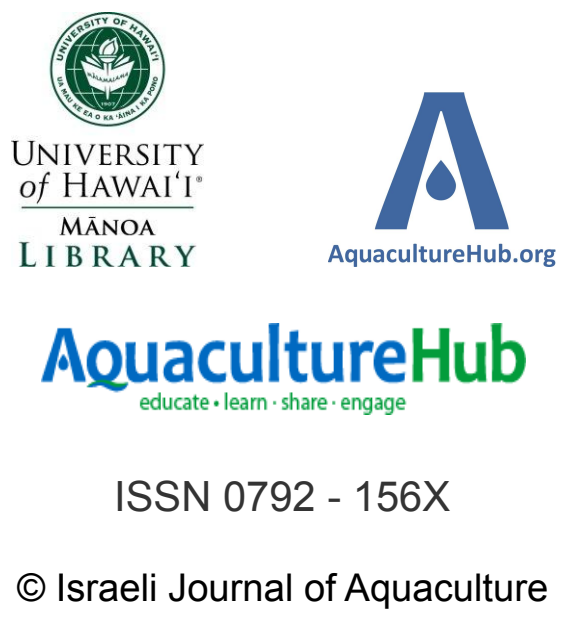



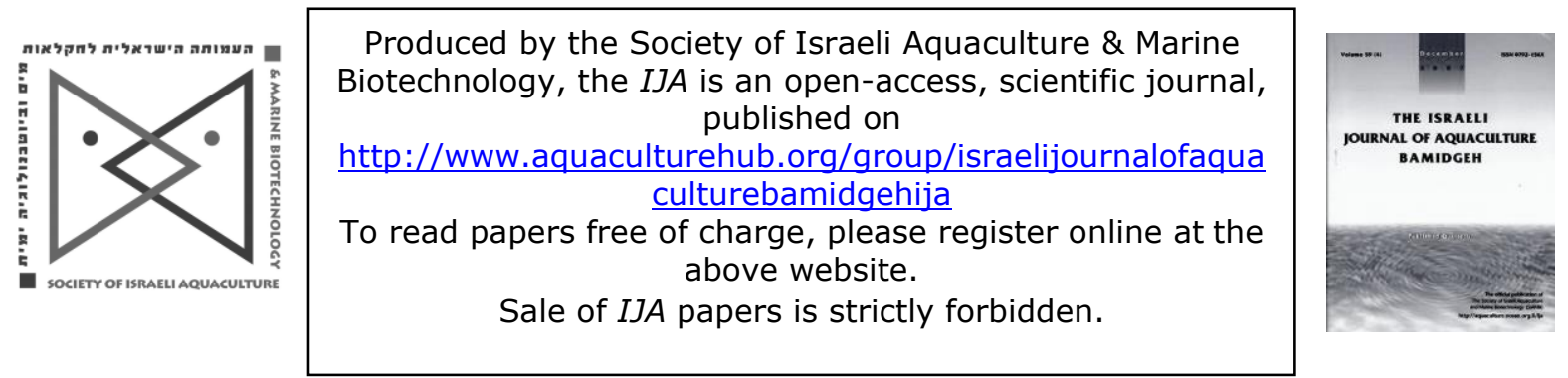

\title{
Depletion Study of Oxolinic Acid in Freshwater Softshell Turtle (Pelodiscus sinensis) with Multiple-Dose Oral Administration
}

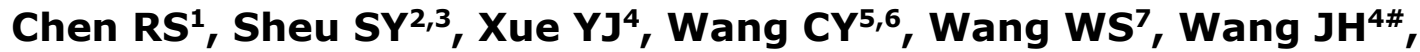 \\ Kuo TF"\# and Chou $\mathrm{CH}^{1 * *}$ \\ ${ }^{1}$ Graduate Institute of Veterinary Medicine, National Taiwan University, Taipei 106, \\ Taiwan \\ 2 School of Medicine, Chung Shan Medical University, Taichung 402, Taiwan \\ ${ }^{3}$ Department of Integrated Chinese and Western Medicine, Chung Shan Medical \\ University Hospital, Taichung 402, Taiwan \\ ${ }^{4}$ Department of Veterinary Medicine, National Chiayi University, Chiayi 600, Taiwan \\ ${ }^{5}$ Department of Cell Biology and Anatomy, College of Medicine, National Cheng Kung \\ University, Tainan 701, Taiwan \\ ${ }^{6}$ Institute of Basic Medical Sciences, College of Medicine, National Cheng Kung University, \\ Tainan 701, Taiwan \\ 7 Department of Post-Baccalaureate Veterinary Medicine, Asia University, Taichung 413, \\ Taiwan
}

Keywords: residues; depletion study; oxolinic acid; softshell turtle

\begin{abstract}
All drugs used in aquaculture must be approved by the government agency responsible for veterinary medicine; prudent use of antibiotics under veterinary supervision is critical in ensuring safety of aquaculture products. The international production of farmed turtle, considered a cultured aquatic species, has been growing continuously but control of bacteria proliferation in turtle farms requires frequent use of antimicrobials. In the present investigation, the residue depletion and withdrawal period of quinolone antibacterial oxolinic acid (OXO) was evaluated in Chinese softshell turtle Pelodiscus sinensis, after repeated (once daily for 5 days) oral doses of medicated feed containing OXO, at the dose of 30 and $60 \mathrm{mg} / \mathrm{kg}$ body weight (bw). Muscle and liver tissues were collected at 1, 2, 4, 8, 12, 16, 20, 24, 28, $32,36,40,44$, and 48 days posttreatment, and OXO was quantified using a validated high-performance liquid chromatography with diode array detector (HPLC-DAD) method. According to the test preparation record, the limit of detection (LOD) and limit of quantification (LOQ) for OXO were estimated at 0.03 and $0.1 \mu \mathrm{g} / \mathrm{g}$, respectively. Considering a maximum residue level (MRL) of $0.1 \mathrm{\mu g} / \mathrm{g}$ for OXO in muscle in European Union (EU) legislative framework, the longest withdrawal period of 72 days was calculated on the basis of a safety span (corresponding to $50 \%$ of the time point when at which residues fall below the MRL) added to the depletion time (48 days). These results may be helpful not only for the establishment of public policies regarding the use of OXO for turtle farming but also to producers for proper handling to ensure safe consumption.
\end{abstract}

\footnotetext{
* Corresponding author. Chung-Hsi Chou, e-mail: cchou@ntu.edu.tw

\# These authors contributed equally to this work
} 


\section{Introduction}

The Chinese softshell turtle (Pelodiscus sinensis), an important member of reptilian aquaculture with high nutritional and medicinal value, is the best-known species in Asia due to its large-scale farming (Rawski et al., 2018). However, literature concerning the residual studies in turtle farming is limited, e.g., ticarcillin (Manire et al., 2005), florphenicol (Zhu et al., 2006), melamine (He et al., 2012), and meloxicam (Lai et al., 2015). Similarly, aquatic animal diseases are a major constraint for increasing aquaculture production. The water used in aquaculture operations provides an environment for growth and proliferation of bacteria which can be influenced by $\mathrm{pH}$, temperature, nutrient availability, and other factors that affect their growth pattern, virulence, and pathogenicity. Most bacteria responsible for causing disease in aquatic animals are gram-negative rods, but some pathogens that are gram-positive rods or cocci and a few that are acid-fast rods also cause disease (Ozturk and Altinok, 2014). Therapeutic regimes are designed to maximize efficacy and thereby minimize the risk of the development of resistant pathogens.

Oxolinic acid (OXO) is a synthetic antibiotic belonging to the quinolone group, which possesses a broad-spectrum antimicrobial agent with bactericidal activity by inhibiting the enzyme DNA gyrase (Wolfson et al., 1989). It is available for clinical use, and veterinary practice to treat parasitic infections because of these characteristics which include high bioavailability, good tissue penetration, long half-lives, high efficacy, and low incidence of adverse effects (Bisacchi, 2015; Naeem et al., 2016). OXO is authorized in veterinary medicine for use in animals including finfish, calves, pigs, and poultry, etc. For pigs and poultry, it was administered at doses of $20 \mathrm{mg} / \mathrm{kg}$ bw/day for up to 5 days, while in finfish it was given orally at doses of $12 \mathrm{mg} / \mathrm{kg}$ bw/day for up to 7 days (EMA, 2005). The European Medicines Agency (EMA) has set the microbiological acceptable daily intakes (ADI) value for OXO at $2.5 \mu \mathrm{g} / \mathrm{kg}$ body weight (i.e., $150 \mu \mathrm{g} /$ person) (EMA, 2005). To protect consumer health, many countries have defined maximum residue limits (MRL) of OXO in animal-derived products (Codex, 2016). The permitted limits the MRLs of OXO in muscle and liver tissues of all species are 0.1 and $0.15 \mu \mathrm{g} / \mathrm{g}$, respectively, stated by the European Union (EU) Commission Regulation 37/2010 (EU, 2009).

The use of OXO in food-producing turtles also has the potential to generate residues/food contamination in muscle tissues and poses a health hazard to the consumer; proper drug administration and establishing the withdrawal period (withholding time) when the animal can be safely slaughtered for food are essential. A number of studies have addressed the pharmacokinetics of OXO in fish farming, such as rainbow trout (O. mykiss), channel catfish (I. punctatus), orange-spotted grouper (E. coioides), Atlantic halibut (H. hippoglossus), corkwing wrasse (S. melops), sea bass ( $D$. labrax), gilthead sea bream (S. aurata), sharpsnout sea bream (D. puntazzo), turbot (S. maximus), cod (G. morhua), kuruma shrimp ( $P$. japonicus), Atlantic salmon (S. salar) etc. Although previous residue reports for OXO have been published (Tyrpenou and Rigos, 2004; Tu et al., 2006), to our knowledge, no previous work has been conducted in freshwater turtles. All prior studies concerning residual control need to define an analytical strategy based on confirmatory methods. High performance liquid chromatography (HPLC) with fluorescence and UV detection is used for analysis in the majority of the methods (Norambuena et al., 2013), while mass spectrometry is used in some laboratories (Bladek et al., 2011).

The purpose of this study was to validate an analytical method and to investigate the tissue distribution and residue depletion of OXO in softshell turtle ( $P$. sinensis) in support of safe approval for food-producing turtle use in Taiwan. It describes a HPLC method with UV detection for OXO residue determination in muscle and liver tissues of cultured softshell turtle. These results make it possible to obtain a product compatible with the legislative framework, with the intention of protecting consumer health and commercialization of this food commodity.

Softshell turtles.

\section{Materials and Methods}

A total of two hundred healthy turtles ( $P$. sinensis) of both sexes, (weight $450 \pm 50 \mathrm{~g}$ ), were used throughout this study. They were obtained from a commercial source and were housed in continuous flow-through tap water system until used for experimentation. All experimental procedures were approved by the Institution of Animal Care and Use 
Committee of National Chiayi University. The turtles were acclimatized for two weeks during which they were offered raw fish and lettuce ad libitum, and maintained in water at a temperature of approximately $22-28^{\circ} \mathrm{C}$, salinity of $39.2-39.7$, and average $\mathrm{pH}$ of $8.05 \pm 0.33$, conditions suitable for the maintenance of aquatic species in a closed system in a tropical area.

Reagents.

HPLC-grade methanol, hexane, and acetonitrile, and reagent-grade oxolinic acid, sodium hydroxide, hydrochloric acid, ammonium hydroxide $\left(\mathrm{NH}_{4} \mathrm{OH}\right)$, sodium dihydrogen phosphate $\left(\mathrm{NaH}_{2} \mathrm{PO}_{4}\right)$, and disodium hydrogen phosphate $\left(\mathrm{Na}_{2} \mathrm{HPO}_{4}\right)$ were used for preparing the solutions or sample treatments. Deionized water was obtained from a Milli$\mathrm{Q}$ Integral system equipped with a $0.22 \mu \mathrm{m}$ membrane point-of-use cartridge.

Preparation of solutions and standards.

$20 \mathrm{mM}$ phosphate buffer solution (PBS) was prepared by dissolving $6.4 \mathrm{~g}$ sodium dihydrogen phosphate and $21.8 \mathrm{~g}$ disodium hydrogen phosphate in $1000 \mathrm{~mL}$ of distilled water. The $\mathrm{pH}$ value was adjusted to 7.4 . Stock solution of oxolinic acid $(1000 \mu \mathrm{g} / \mathrm{mL})$ was prepared by dissolving $25 \mathrm{mg}$ in $25 \mathrm{~mL}$ of methanol and storing in the dark at $0-4^{\circ} \mathrm{C}$. This stock solution was prepared at multilevel working standards with $20 \mathrm{mM}$ PBS $(\mathrm{pH} 7.4)$ that bracketed the intended sample concentration of $0.025,0.05,0.1,0.25$, $0.5,1,2.5,5$ and $10 \mu \mathrm{g} / \mathrm{mL}$.

Pharmacokinetic study.

Animals ( $n=6 /$ group) were randomized divided into treatment groups. They were given OXO in capsule form with a meal once daily for five days to define the optimal dose and dosing interval for the field trial. The low dose of $30 \mathrm{mg} / \mathrm{kg} /$ day was selected based on empirical, positive impressions of clinical efficacy in other aquatic species (Endo et al., 1973; Rigos et al., 2003). The high dose of $60 \mathrm{mg} / \mathrm{kg} /$ day was chosen to monitor safety at the 2 dose levels in multiple-dose study. Six turtles were taken at the following time points: $1,2,4,8,12,16,20,24,28,32,36,40,44$, and 48 days after the last dose for the determination of tissue OXO concentrations. Following completion of muscle and liver sampling, samples were kept frozen at $-20^{\circ} \mathrm{C}$ until analyzed. Animals in the control set (no OXO added) were processed simultaneously.

Extraction \& cleanup process.

Extraction and cleanup which was performed after a previous extraction included the following steps. When muscle or liver samples were thawed and homogenized, sample homogenate $(3.0 \mathrm{~g})$ was weighed in a $15-\mathrm{mL}$ polypropylene centrifuge tube, and $50 \mathrm{mM}$ PBS at pH 7.4 (5 mL) was added. The mixture was then placed in an ultrasonic bath (10 $\mathrm{min})$ and centrifuged $(4,000 \mathrm{xg}, 10 \mathrm{~min})$. The supernatant was then transferred into a clean tube. The extraction procedure was performed twice, and the two extracts were combined. Hexane $(2 \mathrm{~mL})$ was added to the extract sample and the mixture was thoroughly shaken in a vertical shaker for $10 \mathrm{~min}$. Samples were centrifuged $(4,000 \mathrm{xg}$, $10 \mathrm{~min})$, then the upper hexane layer was discarded. The remaining extract in the water layer was collected and saved for later use.

A 3-mL (500 mg) C-18 solid-phase extraction cartridge (Sep-Pak C18) was activated with $3 \mathrm{~mL}$ of methanol followed by $3 \mathrm{~mL}$ of deionized water at the rate of $1 \mathrm{~mL} / \mathrm{min}$. Care was taken to prevent air from reaching the stationary phase in this step. After this muscle or liver sample extraction was loaded onto the column and disposed of. The column was subsequently washed with $3 \mathrm{~mL}$ of deionized water at a rate of $1 \mathrm{~mL} / \mathrm{min}$. Elution was then carried out with $5 \mathrm{~mL}$ of a mixture of methanol and $30 \%$ ammonium hydroxide (74: $26 \mathrm{v} / \mathrm{v})$ at $1 \mathrm{~mL} / \mathrm{min}$ and decanted into a 10- $\mathrm{mL}$ Quickfit glass tube. The extract was evaporated under a gentle stream of nitrogen at $40^{\circ} \mathrm{C}$. The dry residue was dissolved as described above in $50 \mathrm{mM}$ PBS ( $\mathrm{pH} 7.4,1 \mathrm{~mL}$ ) and filtered through a PVDF Millex filter $(0.45 \mu \mathrm{m}, 13 \mathrm{~mm})$. Finally, $20 \mu \mathrm{L}$ was injected into the HPLC system for OXO analysis under the optimized analytical conditions.

Chromatography analysis.

Samples were analyzed in diode array detection HPLC following the analytic method of Tu et al. (2006), with minor modification. Analytes were separated using a C18 reversed-phase column $(150 \times 4.6 \mathrm{~mm}$ ID, $5 \mu \mathrm{m}$ Cosmosil 5C18-MS-II $)$. The waters HPLC system consisted of $600 \mathrm{E}$ multi-solvent delivery system, a 717 auto sampler, and a 996 photodiode array detector set from 210 to $300 \mathrm{~nm}$ to scan the adsorption spectrum of OXO. The injection volume was $20 \mu \mathrm{L}$, while the mobile phase consisted of acetonitrile:water $(50: 50, \mathrm{v} / \mathrm{v})$, an isocratic elution was used at a flow rate of 0.5 
$\mathrm{mL} / \mathrm{min}$.

HPLC methods were validated prior to assay. The intra-day and inter-day precision as well as accuracy of the method were assessed by spiking OXO at three concentrations of $0.1,1$, and $10 \mu \mathrm{g} / \mathrm{mL}$. A triplicate determination of each concentration was conducted during routine operation of the system over a period of three consecutive days. The recovery test was preceded in triplicate by spiking blank tissue homogenates (muscle and liver) with known concentrations $(0.1,1$ and $10 \mu \mathrm{g} / \mathrm{g}$ ) of OXO. Data acquisition was performed using Millennium 32 software (Version 4.0, Waters).

Methods validation

\section{Results}

The following validation parameters were achieved: linearity $(r)$ higher than 0.997 in the range from 0.025 to $10 \mu \mathrm{g} / \mathrm{mL}$, the intra and inter-day precision (CV\%) were lower than 5.2 and $4.2 \%$, respectively. Under the conditions mentioned above, the examined OXO was well resolved and was achieved in less than $8 \mathrm{~min}$. Analyte detection and quantification was realized at $260 \mathrm{~nm}$. The method showed satisfactory recoveries (75 to $88 \%$ ) for all concentrations studied. The limit of detection (LOD) was estimated at 0.03 $\mu \mathrm{g} / \mathrm{g}$ (average blank signal +3 standard deviations of the blank signal), and the limit of quantification (LOQ) was estimated at $0.10 \mu \mathrm{g} / \mathrm{g}$ (average blank signal +10 standard deviations of the blank signal). No effect of the co-extracted biological material was detected.

\section{Residue depletion studies}

The multiple-dose treatment in the residue study was measurable for up to 48 days. After consecutive 5-day oral dosing at 30 or $60 \mathrm{mg} / \mathrm{kg} /$ day, OXO residue concentrations for the high-dose group increased proportionally, calculated as twice that obtained for the group dosed at $30 \mathrm{mg} / \mathrm{kg}$. The results of the OXO depletion are shown in Table 1, indicating that there was accumulation of OXO when given at 30 or $60 \mathrm{mg} / \mathrm{kg}$ for 5 -day and that OXO is slowly eliminated after the discontinuation of the treatment. These data indicated that the concentrations of OXO in the tissues were the highest at day one and declined in the following days to near-undetectable levels: range $0.06-0.10 \mu \mathrm{g} / \mathrm{g}$ in the muscle (at day 44), and $0.05-0.10 \mu \mathrm{g} / \mathrm{g}$ in liver (at day 48), respectively, for animals in the high-dose groups, which were below the MRL values for muscles at $0.1 \mu \mathrm{g} / \mathrm{g}$ by the European Community (EU, 2009). The highest residue concentrations occurred in the liver at all times.

Table 1. Concentrations (Mean $\pm \mathrm{SD}$ ) of oxolinic acid $(\mu \mathrm{g} / \mathrm{g})$ in Chinese softshell turtle (Pelodiscus sinensis) tissues following multiple dosing ( 5 days) of medicated diet ( $30 \& 60 \mathrm{mg} / \mathrm{kg}$ turtle, $\mathrm{n}=6 / \mathrm{group}$ ) at $22-28^{\circ} \mathrm{C}$.

\begin{tabular}{|c|c|c|c|c|c|c|}
\hline \multirow[b]{2}{*}{$\operatorname{Days}^{1}$} & \multicolumn{3}{|c|}{ Low dose $(30 \mathrm{mg} / \mathrm{kg} /$ day $)$} & \multicolumn{3}{|c|}{ High dose $(60 \mathrm{mg} / \mathrm{kg} /$ day $)$} \\
\hline & Control & Muscle & Liver & Control & Muscle & Liver \\
\hline 1 & $\mathrm{UDL}^{2}$ & $3.01 \pm 0.15$ & $3.55 \pm 0.27$ & UDL & $5.71 \pm 0.58$ & $5.21 \pm 0.72$ \\
\hline 2 & UDL & $2.66 \pm 0.28$ & $2.86 \pm 0.25$ & UDL & $4.75 \pm 0.59$ & $4.23 \pm 0.65$ \\
\hline 4 & UDL & $2.29 \pm 0.26$ & $2.24 \pm 0.44$ & UDL & $3.46 \pm 0.31$ & $3.08 \pm 0.44$ \\
\hline 8 & UDL & $2.20 \pm 0.12$ & $1.31 \pm 0.39$ & UDL & $2.24 \pm 0.13$ & $1.79 \pm 0.15$ \\
\hline 12 & UDL & $1.03 \pm 0.09$ & $0.78 \pm 0.16$ & UDL & $1.92 \pm 0.09$ & $1.17 \pm 0.05$ \\
\hline 16 & UDL & $0.61 \pm 0.11$ & $0.56 \pm 0.09$ & UDL & $1.53 \pm 0.11$ & $1.05 \pm 0.03$ \\
\hline 20 & UDL & $0.21 \pm 0.06$ & $0.32 \pm 0.09$ & UDL & $0.62 \pm 0.07$ & $0.80 \pm 0.08$ \\
\hline 24 & UDL & $0.14 \pm 0.03$ & $0.24 \pm 0.04$ & UDL & $0.46 \pm 0.07$ & $0.45 \pm 0.08$ \\
\hline 28 & UDL & $0.13 \pm 0.03$ & $0.16 \pm 0.03$ & UDL & $0.34 \pm 0.08$ & $0.27 \pm 0.04$ \\
\hline 32 & UDL & $0.09 \pm 0.03$ & $0.10 \pm 0.03$ & UDL & $0.23 \pm 0.04$ & $0.23 \pm 0.05$ \\
\hline 36 & UDL & $0.07 \pm 0.02$ & $0.07 \pm 0.02$ & UDL & $0.17 \pm 0.03$ & $0.20 \pm 0.04$ \\
\hline 40 & UDL & $0.06 \pm 0.01$ & $0.06 \pm 0.01$ & UDL & $0.10 \pm 0.03$ & $0.16 \pm 0.04$ \\
\hline 44 & UDL & UDL & $0.05 \pm 0.01$ & UDL & $0.09 \pm 0.02$ & $0.11 \pm 0.03$ \\
\hline 48 & UDL & UDL & UDL & UDL & $0.06 \pm 0.02$ & $0.07 \pm 0.02$ \\
\hline
\end{tabular}

${ }^{1}$ Day 1 is the first day after the final dose.

$2 \mathrm{UDL}=$ Under detection limit.

\section{Discussion}

Literature records concerning the pharmacokinetics of OXO in turtle are currently limited. Previous studies have reported that OXO had a minimum inhibiting concentration (MIC) that ranged between 3.8 to $5.6 \mu \mathrm{g} / \mathrm{mL}$. This concentration can inhibit the growth of major pathogenic bacteria (e.g., $V$. alginolyticus, $V$. harveyi, $V$. campbellii, $V$. parahaemolyticus and $A$. hydrophila) in seawater and freshwater (Li et al., 2001). A toxicological examination of OXO was performed at overdoses $(1600 \mathrm{mg} / \mathrm{kg})$ fifty times 
the standard dose in softshell turtle $(250 \mathrm{~g})$, and surviving animals showed no clinical signs of obvious toxicity 48 hours post-administration (Li et al., 2001).

There have been no reports on residue depletion of OXO in softshell turtle to date. In comparison to other aquatic species testing, a single-dose pharmacokinetics of OXO in orange-spotted grouper (Epinephelus coioides) revealed that the elimination half-life was 15.1 hours in the serum, at an administation dosage $40 \mathrm{mg} / \mathrm{kg}$ at $24 \pm 2{ }^{\circ} \mathrm{C}$ (fish were sampled at 3, 6, 9, 12, 24, 48, 72, 96, 168, 240 and 336 hours after administration), and the residues dropped below the MRL approximately after 10 days following medication (Guo and Liao, 1994).

In the case of OXO, multiple medications, $30 \mathrm{mg} / \mathrm{kg} /$ day for 10 days treatment at 19 $\pm 1^{\circ} \mathrm{C}$ in seawater it was found the accumulation of drugs following multiple dosing is directly proportional to the elimination half-life $(t 1 / 2)$ in association with the dosing interval (Rigos et al. 2003). The calculated $t 1 / 2$ of OXO were estimated to be $11-14$ and 11-19 hours in several tissues, including plasma, muscle, liver, and skin of gilthead seabream and sharpsnout seabream, respectively. The above data revealed that the concentrations of OXO against in the edible tissues were below the MRL at day-11 (24 h post-multiple 10-day dosing), thus, the fast elimination and rapid depletion of OXO suggests short withdrawal times (within $24 \mathrm{~h}$ of cessation of treatments) with reference to human consumption of treated sparids (Rigos et al., 2003). The reason for the apparent discrepancies between in vivo depletion studies is unclear; however, it may relate to differences in species; more work is needed to clarify this issue.

Although there was no information concerning the further metabolism of OXO in sparids, previous rainbow trout and yellowtail studies have demonstrated that glucuronide (OXO-G) was found to be the major metabolite of OXO. Glucuronidation is therefore thought to be the most important stage of the metabolic pathway for OXO in fish (Ishida, 1992; Ueno et al., 1998).

In addition, the elimination rates of antibiotics also depend on several environmental factors such as light, salinity, or seawater temperature. A previous study on the depletion of veterinary drugs in gilthead seabream under experimental field conditions at 14 and $19^{\circ} \mathrm{C}$ (Gonzalez et al., 2010) showed that microbial degradation contributes more to the disappearance of OXO than do environmental factors such as temperature. Additionally, OXO was eliminated much faster in rainbow trout kept in seawater than those in freshwater (Ishida, 1992), the cause of salinity plays an important role in xenobiotic metabolism and excretion.

On the whole, this is the first residual study of OXO in softshell turtle, the final withdrawal period has to be set in a way that the residues in all target tissues drop below their specific MRLs, and, in addition, that the amount of residues in the edible portion drops below the ADI $(2.5 \mathrm{\mu g} / \mathrm{kg}$ bw/day) (EMA, 2018). This means that the longest withdrawal period must be selected to be in full compliance with the MRLs and the ADI. Therefore, to account for the high variability of the residue data, a safety span must be added to the depletion time of 48 days. A safety span corresponding to $50 \%$ of the 48 day depletion time can be seen as appropriate. Thus, the conclusive approach would then result in a withdrawal period of 72 days in the present study.

The present study provides preliminary data supporting more prudent use of OXO for turtle farming and suggests a possible withdrawal time after treatment. The long clearance of OXO from edible tissues may correlate with their slow metabolism (Martin and Palumbi, 1993), but excretion mechanisms remain unclear.

In conclusion, our study is the first to report OXO depletion in freshwater softshell turtle. The potential health risk of turtle dishes to humans, as a consequence of OXO residues derived from turtles treated with medicated feed, in the doses used in this study (30 \& $60 \mathrm{mg} / \mathrm{kg}$ bw for 5-day), can be considered safe for consumption if a withdrawal period of 72 days is observed. The findings suggest that turtles metabolize OXO much more slowly than other aquatic animals studied. The depletion behavior we have detailed in this work could therefore aid in the design and application of new policies of antimicrobial surveillance.

\section{Acknowledgments}

The authors acknowledge the financial support of the Council of Agriculture, Executive Yuan, Taiwan, ROC (Project 102AS-11.3.3-FA-F1(2)). 


\section{References}

Bisacchi, G.S., 2015. Origins of the quinolone class of antibacterials: An expanded "discovery story". J. Med. Chem., 58(12):4874-4882.

Bladek, T., Posyniak, A., Gajda, A., Gbylik, M., Zmudzki, J., 2011. Multi-class procedure for analysis of antibacterial compounds in animal tissues by liquid chromatography-tandem mass spectrometry. Bull. Vet. Inst. Puławy, 55:741-748.

Codex, 2016. Codex Alimentarius Commission. Circular letter 2016/42-RVDF Appendix 1: Database on countries' needs for MRLs.

Endo, T., Ogishima, K., Hatasaka, H., Kaneko, S., Oshima, S., 1973. Application of oxolinic acid as a chemotherapeutic agent against infectious diseases in fishes. 1. Antibacterial activity, chemotherapeutic effects and pharmacokinetic of oxolinic acid in fishes. Bull. Jap. Soc. Sci. Fish, 39(2):165-171.

EMA (European Medicines Agency), 2005. Committee for medical products for veterinary use: Oxolinic acid, Summary report (5), EMEA/CVMP/41090/2005-Final.

EMA (European Medicines Agency), 2018. Committee for medical products for veterinary use: Guideline on determination of withdrawal periods for edible tissues, EMEA/CVMP/ SWP/735325/2012.

EU (European Union), 2009. Commission Regulation No 37/2010 on pharmacologically active substances and their classification regarding maximum residue limits in foodstuffs of animal origin.

Gonzalez, R.R., Fernandez, R.F., Vidal, J.L.M., Muros, M.J.S., Frenich, A.G., 2010. Depletion of veterinary drugs used in aquaculture after administration in feed to gilthead seabream (Sparus aurata). J. Food Prot. 73(9):1664-1670.

Guo, J.J., Liao, I.C., 1994. Pharmacolinetics of oxolinic acid in orange-spotted grouper, Epinephelus coioides, after single oral administration at $24^{\circ} \mathrm{C}$. J. Fish Soc. Taiwan, 21(3):263-272.

He, Z., Zhang, H.Q., Shi, L.K., Liang, L.J., Yao, G.H., 2012. Pharmacokinetic of melamine in tissues from Pelodiscus sinensis. Chin. J. Vet. Sci., 32(2):277-280.

Ishida, N., 1992. Tissue levels of oxolinic acid after oral or intravascular administration to fresh water and sea water rainbow trout. Aquaculture, 102(1-2):9-15.

Lai, O.R., Di Bello, A., Soloperto, S., Freggi, D., Marzano, G., Cavaliere, L., Crescenzo, G., 2015. Pharmacokinetic behavior of meloxicam in loggerhead sea turtles (Caretta caretta) after intramuscular and intravenous administration. J. Wildl. Dis., 51(2):509-512.

Li, J., Wang, Q., Sun, X.T., Ma, X.D., Liu, D.Y., Tong, X., 2001. Application of oxolinic acid against bacterial diseases in aquatic species and the body residues. J. Fish. Sci. China, 8(3):45-49.

Manire, C.A., Hunter, R.P., Koch, D.E., Byrd, L., Rhinehart, H.L., 2005. Pharmacokinetics of ticarcillin in the loggerhead sea turtle (Caretta caretta) after single intravenous and intramuscular injections. J. Zoo Wildl. Med., 36(1):44-53.

Martin, A.P., Palumbi, S.R., 1993. Body size, metabolic rate, generation time, and the molecular clock. Proc. Natl. Acad. Sci. USA, 90(9):4087-4091.

Mast, R.W., Jeffcoat, A.R., Sadler, B.M., Kraska, R.C., Friedman, M.A., 1983. Metabolism, disposition and excretion of [14C]melamine in male Fischer 344 rats. Food Chem. Toxicol., 21(6):807-810.

Naeem, A., Badshah, S.L., Muska, M., Ahmad, N., Khan, K., 2016. The current case of quinolones: Synthetic approaches and antibacterial activity. Molecules, 21(4):268.

Norambuena, L., Gras, N., Contreras, S., 2013. Development and validation of a method for the simultaneous extraction and separate measurement of oxytetracycline, florfenicol, oxolinic acid and flumequine from marine sediments. Mar. Pollut. Bull., 73(1):154-160.

Ozturk, R.C., Altinok, I., 2014. Bacterial and viral fish diseases in Turkey. Turk. J. Fish Aquat. Sci., 14:275-297.

Rawski, M., Mans, C., Kierończyk, B., Świątkiewicz, S., Barc, A., Józefiak, D., 2018. Freshwater turtle nutrition-a review of scientific and practical knowledge. Ann. Anim. Sci., 18(1):17-37. 
Rigos, G., Nengas, I., Alexis, M., Tyrpenou, A.E., Troisi, G.M., 2003. Tissue distribution and residue depletion of oxolinic acid in gilthead sea bream (Sparus aurata) and sharpsnout sea bream (Diplodus puntazzo) following multiple in-feed dosing. Aquaculture, 224(1/4):245-256.

Tu, C.Y., Ho, S.P., Hung, S.W., Chen, B.R., Tsou, L.T., Wang, W.S., 2006. Development of a technique to detect the residue of oxolinic acid in the serum and muscle of Chinese mitten crab, Eriocheir sinensis. J. Food Drug Anal., 14(4):391-397.

Tyrpenou, A.E., Rigos, G., 2004. Determination of oxolinic acid residues in gilthead seabream (Sparus aurata) muscle tissue and plasma by high-performance liquid chromatography. Chromatographia, 60(11/12):657-661.

Ueno, R., Horiguchi, Y., Kubota, S., 1998. Levels of oxolinic acid in cultured yellowtail after oral administration. Nippon Suisan Gakk., 54:479-484.

Wolfson, J.S., Hooper, D.C., Swartz, M.N., 1989. Mechanisms of action and resistance to quinolone antimicrobial agents, Quinolone Antibacterial Agents. American Society for Microbiology, Washington. 5-34 pp.

Zhu, L.M., Yang, X.L., Lin, Q.C., Cai, L.J., Xu, B.Q., Zhang, H.H., 2006. The residues and pharmacokinetic of florfenicol in Trionyx sinensis following intramuscular injection and oral administration. J. Fish China, 30(4):515-519. 